\title{
Efeitos da mobilidade social na saúde mental de adultos: uma revisão sistemática da literatura
}

\author{
Effects of social mobility on adult mental health: \\ a systematic review of the literature
}

\author{
Lenice de Castro Muniz de Quadros ${ }^{1}$ \\ Helen Castillo Laura ${ }^{1}$ \\ Luciana de Avila Quevedo ${ }^{2}$ \\ Denise Petrucci Gigante ${ }^{3}$
}

${ }^{1}$ Centro de Pesquisas Epidemiológicas, Universidade Federal de Pelotas (UFPel). R. Marechal Deodoro 1160/3, Centro. 96020220 Pelotas RS Brasil. lenicemuniz@hotmail.com ${ }^{2}$ Programa de PósGraduação em Saúde e Comportamento, Universidade Católica de Pelotas. Pelotas RS Brasil. ${ }^{3}$ Programa de PósGraduação em Epidemiologia, Universidade Federal de Pelotas (UFPel). Pelotas RS Brasil.

\begin{abstract}
The scope of this study was to identify longitudinal studies evaluating the relationship between social mobility and mental disorders in adults. An electronic review of the literature was conducted in the PubMed/Medline and PsycINFO databases. The bibliographic references of the articles selected for analysis were also examined for eligibility. Cohort studies were selected taking social mobility as exposure category and mental health-related disorders as the outcome. Seven studies were reviewed and their definition and categorization of exposure and outcome were found to be heterogeneous, thus rendering analysis and comparison of the results found in the various studies difficult. Mental health-related disorders were more common in individuals belonging to lower socio-economic classes, regardless of having upward, stable or downward social mobility. Moreover, the influence of individual socio-economic conditions, assessed in adulthood, appears to be greater than the effect of parental economic status on the mental health of individuals. This review indicates that it is possible to find a relationship between socio-economic status during the course of life and mental health in adulthood. However, the direction taken by this relationship remains unclear.
\end{abstract}

Key words Social mobility, Mental health, Psychiatric disorders, Longitudinal studies
Resumo Este estudo teve como objetivo identificar as pesquisas longitudinais que avaliaram a relação entre mobilidade social e transtornos mentais em adultos. Foi realizada uma revisão eletrônica da literatura nas bases de dados PubMed/Medline e PsycINFO, além do rastreamento das referências bibliográficas dos artigos selecionados para análise. Foram selecionados estudos de coorte, tendo como exposição a mobilidade social e os transtornos relacionados à saúde mental como desfecho. A revisão incluiu sete estudos e identificou que há heterogeneidade na definição e na categorização da exposição e do desfecho, dificultando a análise e a comparação dos resultados encontrados nos diferentes estudos. Os transtornos relacionados à saúde mental foram mais comuns em indivíduos das classes socioeconômicas mais baixas, independente de terem mobilidade social ascendente, estática ou descendente. Além disso, a influência das condições socioeconômicas individuais, avaliada na idade adulta, parece ser maior do que o efeito do nível econômico dos pais sobre a saúde mental dos indivíduos. Esta revisão indica que é possível constatar a relação entre a situação socioeconômica ao longo da vida e a saúde mental na idade adulta. No entanto, a direção desta relação não está bem estabelecida.

Palavras-chave Mobilidade social, Saúde mental, Transtornos psiquiátricos, Estudos longitudinais 


\section{Introdução}

Os transtornos mentais envolvem as dimensões econômica, social, política e cultural e se expressam de maneira diferente nas classes sociais e nas relações de gênero ${ }^{1}$. As discussões a respeito da saúde mental são um campo em permanente construção e marcado pela heterogeneidade e pluralidade ${ }^{2}$.

Embora a saúde mental seja tão importante quanto a saúde física para o bem-estar de indivíduos, sociedades e países, e as estimativas de que os transtornos relacionados a ela representem $12 \%$ da carga global de doenças ${ }^{3}$, estudos que avaliaram o efeito da mobilidade social nela, em adultos, de forma longitudinal, ainda são escassos no Brasil e no mundo ${ }^{4-10}$. Esses estudos, capazes de medir de modo objetivo as relações entre mobilidade social e saúde mental ao longo do ciclo vital, são extremamente importantes para o conhecimento e então para o planejamento e a proposição de politicas publicas.

A expressão do caráter social dos transtornos mentais pode ser percebida na desigualdade de sua distribuição entre homens e mulheres e em diferentes classes sociais ${ }^{1}$. Desigualdades em saúde têm sido investigadas por pesquisadores preocupados com a epidemiologia da doença mental ${ }^{11-13}$. Analisando a relação entre classe social e doença, investigadores concluem que quanto mais baixa a classe social, maior o risco de transtorno psiquiátrico $^{14-19}$, sendo que essas desigualdades representam persistente achado na literatura.

Os efeitos das condições socioeconômicas sobre a saúde mental também têm sido investigados através de estudos longitudinais ${ }^{4-10}$ e de análises que incluem a mobilidade social, seja inter ou intrageracional, como um meio de procurar esclarecer os dois conjuntos de explicações, ou seja, investigar a situação socioeconômica como causa ou como consequência dos transtornos mentais ${ }^{4-10}$. Assim, esta revisão teve como objetivo identificar os estudos longitudinais que avaliaram a relação entre mobilidade social e transtornos mentais em adultos.

\section{Metodologia}

Foi realizada uma revisão eletrônica da literatura nas bases de dados PubMed/Medline e PsycINFO, além do rastreamento das referências bibliográficas dos artigos selecionados para análise. A estratégia de busca foi limitada às publicações com investigações originais em humanos desde
1970 até março de 2014. As buscas foram realizadas entre setembro de 2013 e março de 2014 e transferidas para o software EndNote para revisão, leitura de títulos, resumos e seleção de artigos na íntegra.

Os termos utilizados foram: "social mobility", "mental health"; "psychiatric disorder", "mental disorders", "psychic illness", "psychiatric illness"; e, "cohort", "prospective", "follow-up". Assim, a busca foi conduzida incluindo três grupos de termos para identificação dos artigos. No primeiro momento, o termo mobilidade social ("social mobility") foi incluído e, em seguida, foram inseridos os termos relacionados à saúde mental ("mental health", "psychiatric disorder", "mental disorders", "psychic illness", "psychiatric illness"). Finalmente, o terceiro grupo foi adicionado com os termos que restringem o desenho do estudo (longitudinal; cohort; prospective; follow-up). Dentro de cada um desses dois últimos grupos foi utilizado o operador "or", enquanto que entre os três foi utilizado o operador "and".

Para a inclusão dos artigos foram utilizados os seguintes critérios: a) estudos com delineamento longitudinal; b) estudos em que a exposição era mobilidade social, medida através da mudança de renda ou de ocupação; e, c) cujos desfechos poderiam ser: saúde mental, doença psiquiátrica, doença mental, doença psíquica, transtorno mental e transtorno psiquiátrico. Motivos de exclusão após leitura dos resumos foram: a) estudos envolvendo outras patologias; b) estudos com grupos específicos como idosos e outros; e, c) estudos transversais.

Através dessa busca bastante ampla foram localizadas 1.341 publicações. A Figura 1 mostra o número de artigos localizados, a quantidade retirada e alguns motivos de exclusão em cada passo da revisão. Restaram sete publicações para a leitura na íntegra.

\section{Resultados}

Foram selecionados apenas os estudos de coorte, tendo a mobilidade social como exposição e os transtornos relacionados à saúde mental como desfecho.

A revisão incluiu apenas sete estudos que foram publicados desde 1991 até 2013 e provenientes dos seguintes países: Alemanha, Escócia, Nova Zelândia, Inglaterra, Suécia e Brasil. As referências bibliográficas dos sete artigos foram rastreadas e não foram encontradas outras publicações, além daquelas já identificadas. 


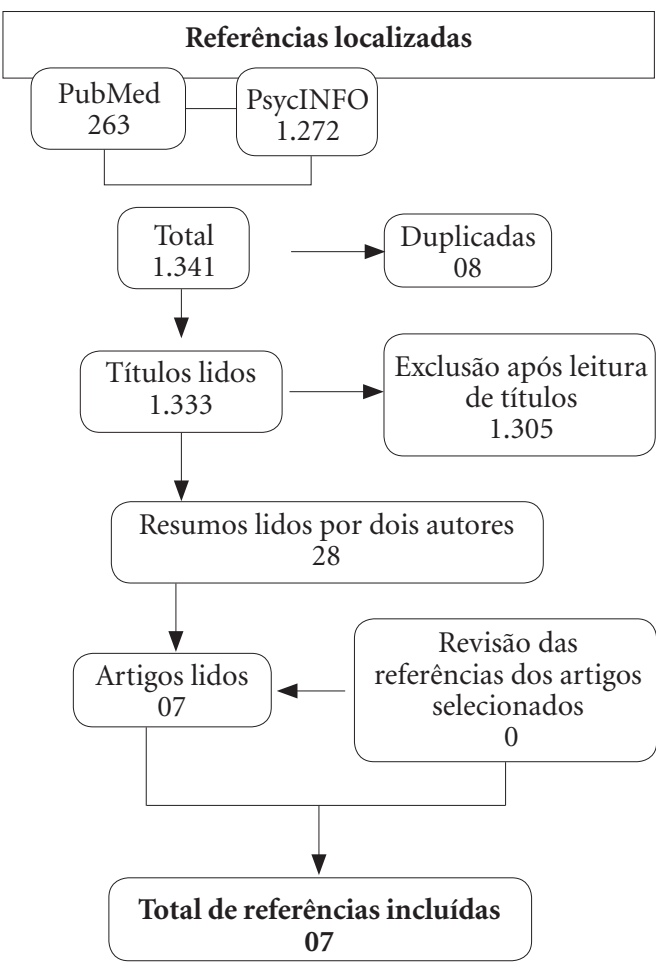

Figura 1. Fluxograma para identificação dos estudos selecionados a partir da busca nas bases de dados.

A descrição dos artigos incluídos nesta revisão, incluindo o nome do primeiro autor, o país onde foi realizado o estudo, o delineamento, o número $(\mathrm{N})$ de participantes, a exposição, o desfecho e as variáveis de ajuste usadas nas análises estatísticas é apresentada no Quadro 1.

Não houve consenso nos critérios utilizados para avaliar a exposição - mobilidade social nem para o desfecho - saúde mental. As definições encontradas nos diferentes estudos tanto para a exposição, como para o desfecho são apresentadas a seguir.

\section{Exposição}

As formas de avaliar a exposição (Quadro 1) foram bastante distintas nos artigos analisados. $\mathrm{O}$ estudo ${ }^{3}$ conduzido na Alemanha, através de entrevistas em dois momentos, avaliou a mudança de classe social que foi determinada de acordo com o modelo de Moore e Kleining que distingue a classe social de acordo com a profissão e a divide em sete categorias. Neste estudo, devido às baixas frequências nas extremidades, a classificação final ficou em quatro classes sociais. Nos três ${ }^{5,6,10}$ estudos realizados na Suécia, a partir de dados oficiais do país, foi utilizada uma classificação baseada na ocupação do entrevistado, embora também faça referência à propriedade do capital e à educação. No estudo realizado na Nova Zelândia ${ }^{7}$, a avaliação socioeconômica foi feita a partir da ocupação, considerando diferentes acompanhamentos e utilizando o nível socioeconômico mais alto investigado dentre os pais do entrevistado e na idade adulta do próprio individuo. $\mathrm{O}$ registro geral de classificação de acordo com o trabalho foi utilizado em estudo conduzido no Reino Unido enquanto a renda familiar em salários mínimos foi a forma utilizada naquele realizado no Brasil ${ }^{9}$.

\section{Desfecho}

As formas de avaliação do desfecho (Quadro 1) foram: entrevista semiestruturada ${ }^{4}$, registros hospitalares conforme convenções do CID 7-9 e CID 8-10,5,10, entrevista diagnóstica, observados os critérios do DSM-IV ${ }^{6}$, Questionário de Saúde Geral (GHQ-28) ${ }^{8}$, e o Self-Report Questionnaire (SRQ-20) ${ }^{9}$.

\section{Fatores de ajuste usados nas análises estatísticas}

As variáveis utilizadas como fatores de ajuste nas análises estatísticas foram: $\mathrm{sexo}^{7,10}$, cor da pele ${ }^{9}$, idade ao diagnóstico ${ }^{10}$, coorte de nascimento $^{10}$, escolaridade materna ${ }^{9}$, classe social dos pais $^{10}$, renda familiar ao nascer ${ }^{9}$, nível socioeconômico na infância ${ }^{7}$, condições de saúde na infância ${ }^{7}$ e transtorno psiquiátrico dos pais ${ }^{10}$.

\section{Discussão}

\section{Estudos que avaliaram o efeito da mobilidade social na saúde mental de adultos}

Essa revisão identificou que há heterogeneidade na definição e na categorização da exposição e do desfecho, dificultando a análise e a comparação dos resultados encontrados nos diferentes estudos.

De acordo com o que foi observado, em todos os estudos incluídos nesta revisão, os transtornos relacionados à saúde mental foram mais comuns em indivíduos das classes socioeconômicas mais 


\begin{tabular}{|c|c|c|c|c|}
\hline Quadro 1. Descr & ão dos estudos sel & cionados para a revisão. & & \\
\hline $\begin{array}{c}\text { Primeiro autor } \\
\text { Ano }\end{array}$ & $\begin{array}{c}\text { Delineamento } \\
(\mathrm{N})\end{array}$ & Exposição & Desfecho & Variáveis de ajuste \\
\hline $\begin{array}{l}\text { Tress } W^{4} \\
1991\end{array}$ & $\begin{array}{l}\text { Estudo de } \\
\text { coorte. } \\
\text { Amostra: } 600\end{array}$ & $\begin{array}{l}\text { Entrevista em dois momentos, classe } \\
\text { social de acordo com o modelo de } \\
\text { Moore e Kleining, segundo a profissão } \\
\text { e divide em } 7 \text { classes. Devido às } \\
\text { baixas frequências nas extremidades a } \\
\text { classificação foi em } 4 \text { classes sociais. }\end{array}$ & $\begin{array}{l}\text { Entrevista } \\
\text { semiestruturada, } \\
\text { anamnese } \\
\text { psicanalítica. }\end{array}$ & - \\
\hline $\begin{array}{l}\text { Timms DWG } \\
1996\end{array}$ & $\begin{array}{l}\text { Estudo de } \\
\text { coorte. } \\
\text { Amostra: } 6.928 \\
\text { homens. }\end{array}$ & $\begin{array}{l}\text { Foram utilizados dados oficiais } \\
\text { do país. Classificação baseada na } \\
\text { ocupação, mas faz referencia também } \\
\text { à propriedade do capital e à educação. }\end{array}$ & $\begin{array}{l}\text { Registros hospitalares } \\
\text { conforme convenções } \\
\text { do CID } 8 .\end{array}$ & - \\
\hline $\begin{array}{l}\text { Timms DWG } \\
1998\end{array}$ & $\begin{array}{l}\text { Estudo de } \\
\text { coorte } \\
\text { Amostra: } \\
15.117\end{array}$ & $\begin{array}{l}\text { Foram utilizados dados oficiais } \\
\text { do país. Classificação baseada na } \\
\text { ocupação, mas faz referencia também } \\
\text { à propriedade do capital e à educação. }\end{array}$ & $\begin{array}{l}\text { Registros hospitalares } \\
\text { conforme convenções } \\
\text { do CID } 8 .\end{array}$ & - \\
\hline $\begin{array}{l}\text { Poulton } \mathrm{R}^{7} \\
2002\end{array}$ & $\begin{array}{l}\text { Estudo de } \\
\text { coorte. } \\
\text { Amostra: } 1000\end{array}$ & $\begin{array}{l}\text { O nível socioeconômico considerou a } \\
\text { ocupação utilizando o patamar mais } \\
\text { alto dentre os pais. }\end{array}$ & $\begin{array}{l}\text { Entrevista diagnóstica } \\
\text { observados os critérios } \\
\text { do DSM-IV. }\end{array}$ & $\begin{array}{l}\text { Sexo } \\
\text { Nível socioeconômico na } \\
\text { infância }\end{array}$ \\
\hline $\begin{array}{l}\text { Tiffin } \mathrm{PA}^{8} \\
2005\end{array}$ & $\begin{array}{l}\text { Estudo de } \\
\text { coorte. } \\
\text { Amostra: } 832\end{array}$ & $\begin{array}{l}\text { Foi utilizado o registro geral de } \\
\text { classificação de acordo com o } \\
\text { trabalho. }\end{array}$ & $\begin{array}{l}\text { Questionário de Saúde } \\
\text { Geral (GHQ-28). } \\
\text { Self-Report }\end{array}$ & - \\
\hline $\begin{array}{l}\text { Anselmi L } 9 \\
2008\end{array}$ & $\begin{array}{l}\text { Estudo de } \\
\text { coorte } \\
\text { Amostra: } 4.297\end{array}$ & Renda familiar em salários mínimos. & $\begin{array}{l}\text { Questionnaire } \\
\text { (SRQ-20). }\end{array}$ & $\begin{array}{l}\text { Cor da pele } \\
\text { Escolaridade materna } \\
\text { Renda familiar ao nascer }\end{array}$ \\
\hline $\begin{array}{l}\text { Tiikkaja S } \\
2013\end{array}$ & $\begin{array}{l}\text { Estudo de } \\
\text { coorte } \\
\text { Amostra: } 1.016 \\
276\end{array}$ & $\begin{array}{l}\text { Foram utilizados dados oficiais } \\
\text { do país. Classificação baseada na } \\
\text { ocupação, mas faz referencia também } \\
\text { à propriedade do capital e à educação. }\end{array}$ & $\begin{array}{l}\text { Registros hospitalares } \\
\text { conforme convenções } \\
\text { do CID 7-9 e CID } \\
\text { 8-10. }\end{array}$ & $\begin{array}{l}\text { Sexo } \\
\text { Idade ao diagnóstico } \\
\text { Coorte de nascimento } \\
\text { Classe social dos pais } \\
\text { Transtorno psiquiátrico } \\
\text { dos pais }\end{array}$ \\
\hline
\end{tabular}

baixas, independente de terem mobilidade social ascendente, estática ou descendente $e^{4-10}$.

As evidências dos estudos revisados sugerem que a importância da influência das condições socioeconómicas do próprio individuo, avaliada na idade adulta, é maior do que o efeito das condições dos pais ${ }^{4,5,9,10}$ sobre a saúde mental desses sujeitos. Já os achados de Poulton et al. ${ }^{7}$ salientam que a mobilidade ascendente desde o nascimento até os 26 anos não atenuou ou reverteu os efeitos adversos do baixo nível socioeconômico na infância sobre a saúde do adulto, sugerindo que a doença mental pode ser consequência da situação social no nascimento.

Um estudo9 ${ }^{9}$ tentou explicar as influências genéticas da doença mental, ajustando para transtorno psiquiátrico parental. No entanto, o ajuste para a história psiquiátrica dos pais tinha apenas um pequeno efeito sobre a associação entre a classe do adulto, a mobilidade social e o risco de transtorno psiquiátrico ${ }^{10}$. $\mathrm{O}$ estudo conclui que independentemente da classe social dos pais, o risco de transtornos psiquiátricos aumenta com maior mobilidade social descendente e diminui 
com o aumento da mobilidade ascendente ${ }^{10}$. $\mathrm{O}$ estudo destaca ainda, que o efeito da mobilidade social por si só aumentando o risco de transtorno psiquiátrico foi significativo entre os homens, mas não entre as mulheres. A ausência de efeito entre as mulheres também pode ser uma questão de poder, uma vez que a maioria dos pacientes psiquiátricos eram homens.

Os membros da coorte provenientes de famílias socioeconomicamente menos favorecidas teriam pior avaliação de sua saúde mental ${ }^{8}$. Esse autor encontrou ainda associação entre queda na trajetória socioeconômica ao longo da vida e pior autoavaliação de saúde mental em homens ( $\mathrm{p}<$ $0,001)$, mas não nas mulheres $(\mathrm{p}=0,8)$, destacando a hipótese de que as condições socioeconômicas ao longo da vida podem agir de forma diferente sobre a saúde mental, dependendo do gênero do entrevistado.

Dificuldades no ambiente familiar ou de trabalho têm sido destacadas como fatores que poderiam estar afetando a maior prevalência de transtornos psiquiátricos em indivíduos pertencentes às classes sociais mais baixas ${ }^{10,20}$. E ainda, a situação socioeconômica dos pais parece mostrar maior influência até a idade em torno de 20 $\operatorname{anos}^{5}$. Depois dessa idade, ou seja, após alcançar a independência, a influência da condição socioeconômica dos pais seria reduzida já que a segurança socioeconômica do próprio sujeito passaria a ter maior importância ${ }^{5}$.

Ao analisar o efeito da mobilidade social intergeracional percebeu-se que o risco de transtorno psiquiátrico diferiu por categorias de classe social dos próprios indivíduos adultos, sem que houvesse influência da dos pais ${ }^{9,10}$. Enquanto a mobilidade social ascendente ou estática foi associada com menor risco de transtorno psiquiátrico, a mobilidade descendente foi associada com risco mais elevado, independentemente da classe social dos pais ${ }^{5,9,10}$.

De acordo com os estudos analisados nesta revisão, foi possível constatar que a mobilidade social descendente esteve associada com piores condições de saúde mental nos estudos incluí$\operatorname{dos}^{5,6,8}$. Algumas hipóteses podem ser levantadas em relação aos resultados aqui apresentados: baixa classe social ao longo da vida pode induzir ao estresse crônico. Este, por sua vez, pode desencadear o desenvolvimento de transtorno psiquiátrico, bem como o baixo nível de educação ou o baixo controle sobre o trabalho ou emprego precário poderiam resultar em formas destrutivas de lidar com estressores.
A preocupação com a associação entre condições socioeconômicas e saúde mental tem sido acompanhada pela discussão sobre a natureza da relação entre os dois conjuntos de conceitos. Para alguns pesquisadores ${ }^{21,22}$, a doença é que pode apresentar efeito sobre as condições socioeconômicas, enquanto outro autor ${ }^{23}$ defende a inversão da cadeia causal, sugerindo que o baixo nível socioeconômico dá origem aos problemas de saúde (seleção social). Esta revisão analisa estudos de coorte como um meio de procurar esclarecer os dois conjuntos de explicações, ou seja, investigar a situação socioeconômica como uma causa ou uma consequência das doenças mentais. No entanto, há poucas evidências quanto a essas hipóteses, possivelmente pelas técnicas de análises dos estudos, principalmente para os mais antigos. Diferenças nas medidas das exposições e dos desfechos e a necessidade de mais estudos, especialmente em países de renda média ou baixa, onde as condições adversas em diferentes momentos da vida ou mesmo intergeracionais são mais frequentes, seriam outros fatores que contribuiriam para que esse conhecimento ainda não esteja completamente estabelecido.

Em síntese, essa revisão indica que é possível constatar a relação entre situação socioeconômica ao longo da vida e a saúde mental na idade adulta. Cabe ressaltar que embora as evidências sejam provenientes de estudos de coorte, alguns desses autores $^{3,4}$ também questionam o sentido dessa associação, sugerindo que a saúde mental tem influência sobre o nível econômico podendo tanto ser causa, como consequência. Apenas um estudo 9 fez a análise longitudinal adequada, considerando as condições socioeconômicas antes do surgimento do desfecho e também ajustando para a saúde mental dos pais. Nesse caso, a conclusão dos autores foi que o risco de transtornos relacionados à saúde mental está inversamente relacionado à classe social. Independentemente da classe social dos pais, o risco cresce com o aumento da mobilidade social descendente e diminui com uma maior mobilidade ascendente. Finalmente, destaca-se que esses estudos, com exceção de um $^{8}$, foram conduzidos em países de renda alta, onde as desigualdades sociais são menos frequentes.

Diante disto recomenda-se o desenvolvimento de estudos multicêntricos que abordem a temática e que utilizem a mesma forma de avaliar a exposição e o desfecho, possibilitando comparações. Sobretudo, recomenda-se que estes estudos sejam desenvolvidos possibilitando a comparação entre países com acentuadas desigualdades sociais. 


\section{Colaboradores}

LCM Quadros e HC Laura trabalharam na concepção, na redação final e na pesquisa e metodologia. LA Quevedo trabalhou na concepção e na redação final. DP Gigante trabalhou na concepção e na redação final e na pesquisa e metodologia.

\section{Referências}

1. Ludemir FM. Desigualdades de classe e gênero e saúde mental nas cidades. Physis 2008; 18(3):451-467.

2. Rinaldi DL. Micropolítica do desejo: a clínica do sujeito na instituição de saúde mental. Cien Saude Colet 2015; 20(2):315-323.

3. Organização Mundial de Saúde (OMS). Relatório sobre a saúde no mundo. Saúde mental: nova concepção, nova esperança. Genebra: OMS; 2001.

4. Tress W, Schwen-Harant T. Psychogenic illness and social mobility between generations. Psychother Med Psychol 1991; 41(1):1-5.

5. Timms DWG. Social mobility and mental health in a Swedish cohort. Soc Psychiatry Psychiatr Epidemiol 1996; 31(1):38-48.

6. Timms D. Gender, Social Mobility And Psychiatric Diagnoses. Soc Sci Med 1998; 46(9):1235-1247.

7. Poulton R, Caspi A, Milde BJ, Thomson WM, Taylor A, Sears MR, Moffitt TE. Association between children's experience of socioeconomic disadvantage and adult health: a life-course study. Lancet 2002; 360(9346):1640-1645.

8. Tiffin PA, Pearce MS, Parker L. Social mobility over the lifecourse and self reported mental health at age 50: prospective cohort study. J Epidemiol Community Health 2005; 59(10):870-872.

9. Anselmi L, Barros FC, Minten GC, Gigante DP, Horta BL, Victora CG. Prevalência e determinantes precoces dos transtornos mentais comuns na coorte de nascimentos de 1982, Pelotas, RS. Rev Saude Publica 2008; 42(Supl. 2):26-33

10. Tiikkaja S, Sandin S, Malki N, Modin B, Sparén P, Hultman CM. Social Class, Social Mobility and Risk of Psychiatric Disorder - A Population- Based Longitudinal Study. PLoS One 2013; 8(11):e77975.

11. Macintyre S. The patterning of health by social position in contemporary Britain: Directions for sociological research. Soc Sci Med 1986; 23(4):393-415.

12. Fox J, editor. Health Inequalities in European Countries. Gower: Aldershot; 1989.

13. Melchior M, Moffitt TE, Milne BJ, Poulton R, Caspi A. Why do children from socioeconomically disadvantaged families suffer from poor health when they reach adulthood? A life-course study. Am J Epidemiol 2007; 166(8):966-974.
14. Dohrenwend BP. Socioeconomic status (SES) and psychiatric disorders: Are the issues still compelling? Soc Psychiatry Psychiatr Epidemiol 1990; 25(1):41-47.

15. Patel V, Araya R, Lima M, Ludermir A, Todd C. Women, poverty and common mental disorders in four restructuring societies. Soc Sci Med 1999; 49(11):1461-1471.

16. Lima MS, Soares BGO, Mari JJ. Saúde e doença mental em Pelotas, RS: dados de um estudo populacional. Rev Psi Clin 1999; 26(5):225-235.

17. Maragno L, Goldbaum M, Gianini RJ, Novaes HMD, Cesar CLG. Prevalência de transtornos mentais comuns em populações atendidas pelo Programa Saúde da Família (QUALIS) no Município de São Paulo, Brasil. Cad Saude Publica 2006; 22(8):1639-1648.

18. Marin-Leon L, Oliveira HB, Barros MB, Dalgalarrondo P, Botega NJ. Social inequality and common mental disorders. Rev Bras Psiquiatr 2007; 29(3):250-253.

19. Lima MCP, Menezes PR, Carandina L, Cesar CLG, Barros MBA, Goldbaum M. Transtornos mentais comuns e uso de psicofármacos: impacto das condições socioeconômicas. Rev Saude Publica 2008; 42(4):717-723.

20. Murali V, Oyebode F. Poverty, social inequality and mental health. Adv Psychiatr Treat 2004; 10:216-224.

21. Illsley R. Occupational class, selection and the production of inequalities in health. Quarterly J. Social Affairs 1986; 2:151-165.

22. West P. Rethinking the health selection explanation for health inequalities. Soc Sci Med 1991; 32(4):373-384.

23. Power C, Manor O, Fox J. Health and Class: The Early Years. London: Chapman and Hall; 1991.

Artigo apresentado em 06/11/2014

Aprovado em 29/04/2015

Versão final apresentada em 01/05/2015 